\title{
Economic-Environmental Planning for Water Quality Control in the Chesapeake Bay Region\#
}

\author{
John H. Cumberland and Henry W. Herzog, Jr.*
}

\section{Introduction}

The quality of the natural environment has become an important determinant in the universal search for the "quality of life". The relationship between this determinant and the monetary component of the "quality of life" is often observed in a sub-national or regional geographic setting where the relationship provides the foundation for the debate between regional economic development and environmental protection.

This study examines one aspect of the debate, namely the conflict between regional economic development and the preservation of regional aquatic resources in one of the largest and most valuable estuarial systems on earth, the Chesapeake Bay region.

The aquatic resources that comprise the Chesapeake Bay region will be subjected to considerable stress in the decades ahead. This stress, specified in terms of potential water quality degradation, is directly related to the failure of regional markets to allocate satisfactorily the limited assimilative resources of the region among competing users. The technological external diseconomies and public good externalities that result whenever resource property rights conflict or are not clearly defined or enforceable cause a divergence between the private and social valuation of water quality services.

For the purpose of this study the inherent difficulty of maintaining water quality and assigning waste treatment responsibilities within the Chesapeake Bay region is termed "the water quality problem". In general, this paper:

(1) defines the dimensions of the water quality problem throughout the Chesapeake Bay region,

(2) forecasts the dimensions of any future stress on the aquatic resources of the region, and

(3) examines the equity and efficiency consequences of alternative management programs for water quality control.

\# The authors thank Professor Gene Bramlett of the University of Georgia for his helpful comments on an earlier version of this paper, and accept responsibility for any remaining shortcomings. The authors also acknowledge with appreciation the financial support which was provided for this research by the Chesapeake Research Consortium, Incorporated, the Office of Water Resources Research of the U.S. Department of the Interior, and by the Bureau of Business and Economic Research of the Division of Behavioral and Social Sciences at the University of Maryland in College Park.

*Professor of Economics, University of Maryland, and Assistant Professor of Economics, University of Tennessee, respectively. 
The regional water quality problem has three major, but inseparable, components. The first of these components involves the generation of waste residuals from the production and consumption of goods and services. Estimates of these residuals, as well as the demographic and economic activities from which they are derived are provided below in Section I.

The aquatic environment comprises the second component of the water quality problem. Here, residuals from the first component, perhaps after change in form or amount, are diluted, transported, transformed, and in some instances, combined with other residual types. The output of this natural process forms the third component, "environmental quality", which may be measured by ambient concentrations, species populations, or aesthetic effects.

Each of the three components must be considered explicitly within any water quality management program for the Chesapeake Bay region. Several management programs that recognize these interdependencies will be examined below in Section II.

\section{Demographic, Economic, and Water Residual Projections for the Chesapeake Bay Region}

Baseline and projected demographic, economic, and residuals generation information for the Chesapeake Bay region will serve as an example of the nature and magnitude of the waste residuals management problem within the natural boundaries of a large water resource system. This information, for both political and river basin "sub-regions", will provide a significant measurement of the critically important interface between man and his regional environment.

For the purpose of this study the Chesapeake Bay region includes the District of Columbia, Baltimore City, all counties in Maryland and seventy-three counties and independent cities in Virginia. Minor civil divisions are the primary areal units within the study. These divisions represent election districts in Maryland and magisterial districts in Virginia. Aggregate information for river basins within the Bay region was obtained by summing over the set of minor civil divisions that most closely delimit the natural drainage areas of the basins.

The construction of both baseline and forecasted demographic, economic, and waste residual information series at the river basin level is a fundamental prerequisite for water resource planning. The importance of natural resource and environmental planning at the river basin level, and thus the desirability of data availability within resource system boundaries rather than political boundaries, have received much attention in the recent literature on water quality management. For this reason, information for the study was accumulated at the minor civil division level (the smallest political unit for which census data are available throughout the region) and subsequently combined to form natural drainage areas.

Six types, or measures, of waterborne waste residuals were considered. These are suspended and dissolved solids, biochemical and chemical oxygen demand, and the nutrients phosphorus and nitrogen. Two of these residuals, biochemical 
oxygen demand and nitrogen, will serve as examples throughout the paper. Denoted "gross water residuals" below, these measures of environmental demand represent the total waste, of a specific type, generated within a minor civil division, county, independent city, state, or river basin prior to treatment or recycling.

For the purpose of this study the generation of each of these residuals is tied to the magnitude of various demographic and economic activities. These activities were selected to attain a maximum coverage of the primary sources of waterborne residuals, namely industrial production, agriculture, and domestic sewage.

Waste residuals from various industrial sources within the Chesapeake Bay region are tied to the output levels (measured in dollars) of specific industries. The coefficients that provide the link between these economic outputs and the waste residuals generated during their creation are termed "gross residual coefficients". These coefficients have been developed for ninety-two industry sectors defined by Standard Industrial Classification (SIC) codes. ${ }^{1}$ The industry definitions and gross residual coefficients for biochemical oxygen demand and nitrogen are presented in Table 1.

The gross residuals that result from domestic sewage are linked to the population level. Runoff from agricultural land is related to irrigated acreage. The gross residual coefficients for domestic sewage and agricultural runoff are defined and estimated in the same way as the industry coefficients, namely tons of residual per person or irrigated acre.

Estimates of gross residual for each waste type, year, and sub-area within the Chesapeake Bay region depend on the gross residual coefficients and the levels of the activities to which each coefficient is tied. The coefficients are assumed to remain constant over the 1970-1985 projection period. Industry outputs and population were forecast at the county level for each county within the Bay region." Irrigated acreage was assumed to remain constant throughout the projection period. ${ }^{3}$

In order to compile baseline and projected gross residual information for the river basins within the Chesapeake Bay region, county-level industrial outputs, population, and irrigated acreage must first be allocated to the constituent minor civil divisions. Since economic and demographic information is available for these divisions only in the base year, 1970, minor civil divisions - county share coefficients for each industry output category, population, and irrigated acreage were computed for 1970 and held constant throughout the projection period. ${ }^{4}$

The share coefficients for industry outputs are equal to the ratio of employees of each sector who reside within a minor civil division to those who reside within the parent county. Because these share coefficients are based on information compiled at worker residences rather than places of employment, the sectoral outputs, and the residual loadings derived from these outputs, are subject to the usual criticisms reserved for regional information obtained under the influence of the "situs problem". The share coefficients for population are obtained in a like fashion. Since no information on irrigated acreage exists at the minor civil division level, the share coefficient for "crops" (industry sector number two in Table 1) was used over the projection period. 
TABLE 1

Industry Sectors and Related Gross Residual Coefficients*

Gross Residual

Industry Sectors:

Coefficients:**

\begin{tabular}{|c|c|c|c|c|}
\hline No. & Name: & SIC Codes $(\mathrm{s}):$ & BOD & Nitrogen \\
\hline 1 & Livestock & Part 01, Part 02 & 1.14622 & .29680 \\
\hline 2 & Crops & Part 01, Part 02 & .00013 & 17298 \\
\hline 3 & Forestry \& Fishery Products & 08,09 & .00014 & .00000 \\
\hline 4 & Agricultural Services & $071,072,073,074$ & .00028 & .00000 \\
\hline 5 & Iron Ore Mining & 101,106 & .00000 & .00000 \\
\hline 6 & Non-Ferrous Ore Mining & $102,103,104,105,108,109$ & .00015 & .00000 \\
\hline 7 & Coal Mining & 11,12 & .00016 & .00000 \\
\hline 8 & Petroleum Mining & 131,132 & .00000 & .00000 \\
\hline 9 & Minerals Mining & $141,142,144,145,148,149$ & .00000 & .00000 \\
\hline 10 & Chemical Mining & 147 & .00000 & .00000 \\
\hline 11 & New Construction & 138, Part 15, Part 16, Part 17 & .00040 & .00000 \\
\hline 12 & Maintenance Construction & Part 15, Part 16, Part 17 & .00006 & .00000 \\
\hline 13 & Ordnance & 19 & .00019 & .00000 \\
\hline 14 & Meat Packing & 201 & .02763 & .00000 \\
\hline 15 & Dairy Products & 202 & .04533 & .00000 \\
\hline 16 & Canned \& Frozen Foods & 203 & .05555 & .00000 \\
\hline 17 & Grain Mill Products & 204 & .00009 & .00000 \\
\hline 18 & Bakery Products & 205 & .00023 & .00000 \\
\hline 19 & Sugar & 206 & .00008 & .00000 \\
\hline 20 & Candy & 207 & .00024 & .00000 \\
\hline 21 & Beverages & 208 & .02180 & .00000 \\
\hline 22 & Misc. Food Products & 209 & .00008 & .00000 \\
\hline 23 & Tobacco & 21 & .00000 & .00000 \\
\hline 24 & Fabrics \& Yarn & $221,222,223,224,226,228$ & .04363 & .00000 \\
\hline 25 & Rugs, Tire Cord, Misc. Textiles & 227,229 & .00000 & .00000 \\
\hline 26 & Apparel & $225,23,3992,-239$ & .00032 & .00000 \\
\hline 27 & Household Textiles \& Upholstery & 239 & .00024 & .00000 \\
\hline 28 & Lumber \& Prod. Exc. Containers & $24,-244$ & .00022 & .00000 \\
\hline 29 & Wooden Containers & 244 & .00015 & .00000 \\
\hline 30 & Household Furniture & 251 & .00033 & .00000 \\
\hline 31 & Office Furniture & $25,-251$ & .00037 & .00000 \\
\hline 32 & Paper \& Prod. Exc. Containers & $26,-265$ & .07804 & .00000 \\
\hline 33 & Paper Containers & 265 & .00024 & .00000 \\
\hline 34 & Printing \& Publishing & 27 & .00019 & .00000 \\
\hline 35 & Basic Chemicals & $281,286,287,289$ & .00018 & .00000 \\
\hline 36 & Plastics \& Synthetics & 282 & .00800 & .00000 \\
\hline 37 & Drugs, Cleaning \& Toilet Items & 283,284 & .00016 & .00000 \\
\hline 38 & Paint \& Allied Products & 285 & .00016 & .00000 \\
\hline 39 & Petroleum Refining & 29 & .00014 & .00000 \\
\hline 40 & Rubber \& Plastic Products & 30 & .00021 & .00000 \\
\hline 41 & Leather Tanning & 311,312 & .08761 & .00000 \\
\hline 42 & Shoes \& Other Leather Prods. & $31,-311,-312$ & .00048 & .00000 \\
\hline 43 & Glass \& Glass Products & $321,322,323$ & .00012 & .00000 \\
\hline 44 & Stone \& Clay Products & $324,325,326,327,328,329$ & .00012 & .00000 \\
\hline
\end{tabular}

*Sector definition from the Regional Forecasting Project, Bureau of Business and Economic Research, University of Maryland. Gross residual coefficients from Robert J. Korbach, An Environmental Linkages Model of the State of Maryland, Bureau of Business and Economic Research, University of Maryland, June, 1972.

**Thousand tons per million dollars of output (in 1970 dollars). 
Gross Residual

Coefficients:

\begin{tabular}{|c|c|c|c|c|}
\hline No. & Name: & SIC Codes $(\mathrm{s}):$ & $\mathrm{BOD}$ & Nitrogen \\
\hline 45 & Iron \& Steel & $331,332,3391,3399$ & .00022 & .00000 \\
\hline 46 & Copper & $3331,3351,3362$ & .00008 & .00000 \\
\hline 47 & Aluminum & $3334,3352,3361$ & .00012 & .00000 \\
\hline 48 & Other Non-Ferrous Metals & $\begin{array}{l}3332,3333,3339,334,3356,3357 \\
\quad 3369,3392\end{array}$ & .00010 & .00000 \\
\hline 49 & Metal Containers & 341,3491 & .00005 & .00000 \\
\hline 50 & Heating, Plumbing, Struc. Metal & 343,344 & .00014 & .00000 \\
\hline 51 & Stamping, Screw Machine Prod. & 345,346 & .00011 & .00000 \\
\hline 52 & Hardware, Plating, Wire Prod. & $342,347,348,349,-3491$ & .00019 & .00000 \\
\hline 53 & Engines \& Turbines & 351 & .00000 & .00000 \\
\hline 54 & Farm Machinery \& Equipment & 352 & .00000 & .00000 \\
\hline 55 & Construction \& Mining Mach. & $3531,3532,3533$ & .00018 & .00000 \\
\hline 56 & Material Handling Equipment & $534,3535,3536,3537$ & .00015 & .00000 \\
\hline 57 & Metalworking Mach. \& Equip. & 354 & .00021 & .00000 \\
\hline 58 & Special Industry Machinery & 355 & .00015 & .00000 \\
\hline 59 & General Industrial Machinery & 356 & .00016 & .00000 \\
\hline 60 & Machine Shops \& Misc. Mach. & 359 & .00022 & .00000 \\
\hline 61 & Office \& Computing Machines & 357 & .00010 & .00000 \\
\hline 62 & Service Industry Machines & 358 & .00013 & .00000 \\
\hline 63 & Electric Apparatus \& Motors & 361,362 & .00025 & .00000 \\
\hline 64 & Household Appliances & 363 & .00017 & .00000 \\
\hline 65 & Electric Light \& Wiring Equip. & 364 & .00029 & .00000 \\
\hline 66 & nication Equipment & 365,366 & .00021 & .00000 \\
\hline 67 & ic Components & 367 & .00032 & .00000 \\
\hline 68 & Batteries \& Engine Elec. Equip. & 369 & .00025 & .00000 \\
\hline 69 & Motor Vehicles & 371 & .00047 & .00000 \\
\hline 70 & Aircraft \& Parts & 372 & .00017 & .00000 \\
\hline 71 & Ships, Trains, Trailers, Cycles & $373,374,375,379$ & .00025 & .00000 \\
\hline 72 & Instruments \& Clocks & $381,382,384,387$ & .00018 & .00000 \\
\hline 73 & Optical \& Photographic Equip. & $383,385,386$ & .00032 & .00000 \\
\hline 74 & Misc. Manufacturing Products & $39,-3992$ & .00026 & .00000 \\
\hline 75 & Transportation & $40,41,42,44,45,46,47$ & .00020 & .00000 \\
\hline 76 & Communication & $481,482,489$ & .00020 & .00000 \\
\hline 77 & Radio, TV Broadcasting & 483 & .00014 & .00000 \\
\hline 78 & Electric Utility & 491,4931 & .00007 & .00000 \\
\hline 79 & Gas Utility & 492,4932 & .00013 & .00000 \\
\hline 80 & Water Utility & $494,495,496,497$ & .00005 & .00000 \\
\hline 81 & Wholesale \& Ret & $50,52,53,54,55,56,57,58,59$ & .00048 & .00000 \\
\hline 82 & Finance \& Insurance & $60,61,62,63,64,66,67$ & .00022 & .00000 \\
\hline 83 & Real Estate \& Rental & $65,-654$ & .00002 & .00000 \\
\hline 84 & Hotels, Personal \& Repair Svc. & $70,72,76,-7694,-7699$ & .03093 & .00000 \\
\hline 85 & Business Services & $654,73,7694,7699,81,89,-736$ & .00012 & .00000 \\
\hline 86 & Automobile Repair Services & 75 & .00006 & .00000 \\
\hline 87 & Amusements \& Recreation & 78,79 & .00018 & .00000 \\
\hline 88 & Medical \& Educational Instit. & $736,80,82,84,86,892$ & .00179 & .00000 \\
\hline 89 & Business Travel, Entertainment & & .00000 & .00000 \\
\hline 90 & Office Supplies & & .00000 & .00000 \\
\hline 91 & 'Government Industry' & & .00036 & .00000 \\
\hline 92 & 'Domestic Service Industry' & & .00186 & .00000 \\
\hline
\end{tabular}


Once sectoral outputs and population have been forecast for each county within the Bay region, these figures can be apportioned to the constituent minor civil divisions using the share coefficients just described. If sectoral output and population information is desired at the river basin level, this information can be obtained by summing over the set of minor civil divisions that comprise each basin. The results of this procedure for the base year, 1970, are presented in Table 2.

Gross residual estimates for counties, minor civil divisions, and river basins can be obtained by first multiplying the gross residual coefficients times their appropriate industry output, population, or acreage, and then summing over like residuals. ${ }^{5}$ Two gross residual projections for the Chesapeake Bay region are presented, by river basins, in Tables 3 and 4. The average annual growth rates provided in the right hand column of each table facilitate comparisons between present and projected levels of nitrogen and biochemical oxygen demand.

Notice that gross nitrogen generation (Table 3) is forecast to increase in each of the Chesapeake Bay river basins over the period 1970-1985, while gross biochemical oxygen demand (Table 4) is projected to decrease in most of the Maryland river basins over the same period. The fact that the latter residual is forecast to decrease within most Maryland river basins is related to the projected decline of livestock production (sector number one in Table 1) in those basins. When applied to the very large gross residual coefficient for this industry (Table 1) the decline in bio-chemical oxygen demand from this one sector more than offsets any projected increase in this same residual from other sources.

The selection of the minor civil division as the primary areal unit in the study provides great flexibility for the aggregation of demographic, economic, and waste residual information to either political or natural system boundaries (such as the river basin). Thus, information such as that provided in Tables 2 through 4 may be examined for any specific minor civil division, independent city, county, state, or group of minor civil divisions desired. This aggregation flexibility is a fundamental prerequisite for efficient regional economic-environmental planning.

However, estimates of gross waste residual generation only serve to quantify the first component of the regional "water quality problem". A micro-economicenvironmental approach is required for a full consideration of both the "problem" and its control through efficient water quality management in the Chesapeake Bay region.

\section{A Simulation of Water Quality Management within the Patuxent River Basin}

The "micro-aspects" of water quality control in the Chesapeake Bay region can be examined through a simulation experiment in one of Bay's major river basins, the Patuxent. The primary goal of this latter section of the study is to demonstrate the economic consequences, in terms of regional efficiency and equity, of alternative water quality management programs. ${ }^{6}$

The Patuxent River is the largest of Maryland's intrastate rivers. Located between the metropolitan centers of Washington, D.C. and Baltimore, this river 
TABLE 2

Industry Outputs and Population in 1970 by Major River Basins within the Chesapeake Bay Region (Outputs are in Millions of 1970 Dollars, Population is in Thousands)

RIVER BASINS

\begin{tabular}{|c|c|c|c|c|c|c|c|c|c|c|c|c|c|c|c|c|}
\hline Ind. Nos. & 1 & 2 & 3 & 4 & 5 & 6 & 7 & 8 & 9 & 10 & 11 & 12 & 13 & 14 & 15 & \\
\hline 1 & .5 & 7.9 & 23.7 & 9.0 & 75.1 & 6.7 & 13.1 & 14.8 & 17.7 & 135.1 & 37.7 & 20.7 & 28.0 & 8.0 & 71.0 & \\
\hline 2 & 1.9 & 12.8 & 19.3 & 6.6 & 14.2 & 5.9 & 5.5 & 34.0 & 8.5 & 61.7 & 5.3 & 9.6 & 6.8 & 7.2 & 53.5 & \\
\hline 3 & .1 & .6 & 13.6 & .0 & 7.7 & 2.7 & .0 & .1 & 1.0 & 16.0 & 8.5 & .4 & 18.2 & .9 & 10.3 & \\
\hline 4 & .1 & .2 & .6 & 5.9 & 10.3 & .4 & 2.5 & 14.5 & 1.3 & 35.2 & 3.5 & 1.8 & 3.8 & .6 & 20.7 & \\
\hline 5 & .0 & .0 & .0 & .0 & .0 & .0 & .0 & .0 & .0 & .0 & .0 & .0 & .0 & .0 & .1 & \\
\hline 6 & .0 & .0 & .2 & .0 & .4 & .0 & .0 & .1 & .0 & .4 & .0 & .0 & .0 & .0 & .2 & \\
\hline 7 & .0 & .0 & .0 & .0 & .2 & .0 & .0 & .0 & .0 & 4.0 & 4.9 & .0 & .0 & .0 & .0 & \\
\hline 8 & .0 & .0 & .1 & .0 & .6 & .0 & .0 & .0 & .0 & .3 & .0 & .0 & .0 & .0 & .2 & \\
\hline 9 & .0 & .0 & 1.3 & 4.8 & 16.5 & .0 & 1.0 & 4.1 & .0 & 33.0 & 2.7 & .0 & .9 & 8.3 & 8.6 & \\
\hline 10 & .0 & .0 & .0 & .0 & .0 & .0 & .0 & .0 & .0 & .0 & .0 & .0 & .0 & .0 & .0 & \\
\hline 11 & .3 & 2.4 & 6.2 & 30.2 & 192.1 & 1.1 & 41.9 & 65.2 & 2.4 & 522.2 & 13.1 & 6.9 & 25.2 & 4.1 & 383.6 & \\
\hline 12 & .2 & 1.8 & 5.7 & 27.5 & 138.0 & 1.4 & 34.5 & 69.2 & 3.2 & 713.6 & 8.9 & 10.1 & 29.7 & 2.8 & 398.1 & \\
\hline 13 & .0 & .0 & .0 & 1.1 & .0 & .0 & 1.4 & .0 & .0 & 10.4 & .0 & .0 & .0 & .6 & 12.0 & \\
\hline 14 & .0 & 6.3 & 43.6 & 2.2 & 344.7 & 10.5 & 6.6 & 5.1 & 29.0 & 42.0 & 18.8 & 28.8 & .0 & .0 & 316.1 & \\
\hline 15 & .1 & .0 & 1.4 & .8 & 40.0 & .3 & 3.4 & 7.2 & 1.5 & 201.4 & 4.8 & .0 & 1.3 & 2.9 & 111.3 & \\
\hline 16 & 1.4 & 6.3 & 24.3 & 2.1 & 60.8 & 6.4 & 4.6 & .3 & 3.0 & 16.6 & 12.9 & 25.4 & 7.3 & .4 & 70.0 & \\
\hline 17 & .0 & .0 & .0 & .9 & 2.6 & 2.7 & 1.9 & .0 & 4.4 & 37.0 & 8.0 & 31.4 & 5.3 & .0 & 30.6 & \\
\hline 18 & .0 & .0 & .0 & .2 & 35.7 & .2 & .4 & 2.7 & .2 & 41.6 & .3 & 2.2 & .3 & .0 & 75.7 & \\
\hline 19 & .0 & .0 & .0 & .0 & .0 & .0 & .0 & .0 & .0 & .0 & .0 & .0 & .0 & .0 & 39.6 & \\
\hline 20 & .0 & .0 & .2 & .1 & 59.1 & .0 & .3 & .3 & .1 & 3.5 & 1.3 & .0 & 1.2 & .2 & 7.2 & \\
\hline 21 & .0 & .0 & .1 & 10.6 & 56.1 & .5 & 28.2 & 9.1 & .4 & 82.1 & 1.2 & 4.4 & 2.6 & .2 & 246.0 & \\
\hline 22 & .1 & .0 & .9 & 1.3 & 38.6 & .2 & 3.9 & 6.6 & .9 & 62.1 & 1.6 & .0 & 1.7 & 2.0 & 117.3 & \\
\hline 23 & .0 & .0 & .0 & .0 & 2888.1 & .0 & 0. & .0 & .0 & 2.9 & 3.4 & .0 & 2.5 & .0 & 1.9 & \\
\hline 24 & .0 & .0 & .0 & 1.5 & 7.1 & .0 & 3.5 & .0 & .0 & 11.8 & 5.8 & .0 & 4.5 & .0 & 17.4 & \\
\hline 25 & .0 & .0 & .0 & .0 & 24.3 & .0 & .0 & .0 & .0 & .0 & .0 & .0 & .0 & .0 & 14.0 & \\
\hline 26 & .1 & 1.1 & 12.5 & 3.3 & 54.7 & 4.0 & 7.5 & .2 & 3.2 & 45.8 & 27.8 & 11.8 & 5.0 & 5.3 & 196.1 & \\
\hline 27 & .0 & .0 & .0 & 1.4 & 19.5 & .0 & 3.9 & 3.6 & .0 & 1.6 & .0 & .0 & .0 & .0 & 23.5 & $y$ \\
\hline 28 & .0 & .1 & 1.2 & 3.0 & 87.7 & 3.3 & 5.9 & 6.1 & 2.6 & 50.4 & 28.0 & 7.6 & 51.0 & .0 & 72.6 & న \\
\hline 29 & .0 & .0 & .0 & 1.0 & 3.7 & .4 & 1.6 & .0 & .1 & .1 & .3 & 1.0 & .9 & 1.5 & 7.6 & $\theta$ \\
\hline 30 & .0 & .8 & .1 & .4 & 13.5 & .0 & .6 & 1.2 & .0 & 12.4 & .0 & .0 & .0 & .0 & 19.1 & 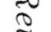 \\
\hline 31 & .0 & .8 & .1 & .0 & 17.7 & .0 & .0 & 1.1 & .0 & 6.2 & 6.8 & .0 & .6 & .0 & 31.6 & \\
\hline 32 & .0 & .2 & .0 & 1.6 & 181.0 & .0 & 1.9 & .4 & .0 & 96.7 & .3 & .0 & 34.2 & .0 & 62.9 & 2 \\
\hline 33 & .3 & .0 & 3.4 & 7.8 & 35.3 & .7 & 11.8 & 1.9 & .0 & 7.0 & .0 & .0 & .0 & .0 & 105.3 & 으 \\
\hline & & & & $\begin{array}{r}1 \\
2 \\
3 \\
4 \\
5 \\
6 \\
7 \\
15\end{array}$ & $\begin{array}{l}\text { Blackwater } \\
\text { Chester } \\
\text { Choptank } \\
\text { Gunpowder } \\
\text { James } \\
\text { Nanticoke } \\
\text { Patapsco } \\
\text { Chesapeake }\end{array}$ & RIVEI & $\begin{array}{r}\text { ASIN } \\
8 \mathrm{P} \\
9 \mathrm{P} \\
10 \mathrm{P} \\
11 \mathrm{R} \\
12 \mathrm{~W} \\
13 \mathrm{Y} \\
14 \mathrm{E} \\
\text { Atlant }\end{array}$ & $\begin{array}{l}Y \\
\text { rent } \\
\text { noke } \\
\text { nac } \\
\text { ahanr } \\
\text { mico }\end{array}$ & & & & & & & & 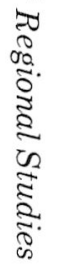 \\
\hline
\end{tabular}


TABLE 2 (continued)

Industry Outputs and Population in 1970 by Major River Basins within the Chesapeake Bay Region (Outputs are in Millions of 1970 Dollars, Population is in Thousands)

Ind. Nos.
34
35
36
37
38
39
40
41
42
43
44
45
46
47
48
49
50
51
52
53
54
55
56
57
58
59
60
61
62
63
64
65
66

RIVER BASINS

\begin{tabular}{|c|c|c|c|c|c|c|c|c|c|}
\hline 3 & 4 & 5 & 6 & 7 & 8 & 9 & 10 & 11 & 12 \\
\hline 17.8 & 5.2 & 107.9 & 1.2 & 15.7 & 17.4 & 1.0 & 500.2 & .4 & 2.5 \\
\hline .2 & 4.6 & 90.9 & .0 & 8.0 & 1.0 & .0 & 18.7 & .7 & .0 \\
\hline .0 & .0 & 404.9 & .0 & 12.0 & 9.3 & .0 & 75.4 & 26.0 & .0 \\
\hline .0 & 8.3 & 73.0 & .0 & 14.4 & 5.3 & .0 & 15.7 & .0 & .0 \\
\hline .0 & .0 & 7.0 & .0 & .0 & 1.5 & .0 & 3.1 & .0 & .0 \\
\hline .1 & .0 & .0 & .0 & .0 & 11.5 & .0 & 30.9 & .0 & .0 \\
\hline 2.8 & 5.1 & 17.9 & 1.5 & 20.8 & .2 & .0 & 68.7 & .0 & .0 \\
\hline .0 & .0 & .4 & .0 & .0 & .0 & .0 & 1.1 & .0 & .0 \\
\hline .0 & .3 & 21.7 & .0 & 3.8 & .0 & .0 & 19.8 & 3.2 & .0 \\
\hline .1 & .0 & .0 & .0 & .0 & .7 & .0 & 39.6 & .0 & .0 \\
\hline .2 & 3.6 & 34.3 & .0 & 14.1 & 12.9 & .0 & 94.6 & 3.5 & .0 \\
\hline .0 & 34.1 & 1.4 & .0 & 45.3 & 1.5 & .0 & 7.9 & .0 & .0 \\
\hline .0 & .0 & 34.0 & .0 & 22.4 & 7.9 & .0 & 1.8 & .0 & .0 \\
\hline .0 & 4.2 & 77.3 & .0 & 5.6 & .0 & .0 & 13.7 & .0 & .0 \\
\hline .0 & .0 & 2.6 & .0 & .0 & 2.5 & .0 & 2.5 & .0 & .0 \\
\hline 1.8 & 2.3 & 3.5 & .1 & 3.1 & .0 & .0 & .1 & .0 & .0 \\
\hline .0 & 1.9 & 45.4 & .1 & 8.0 & 10.6 & .3 & 82.9 & 6.9 & 2.3 \\
\hline .0 & .0 & .0 & .0 & .2 & .3 & .0 & 10.1 & .0 & .0 \\
\hline 8.6 & 1.2 & 21.2 & .6 & 1.6 & .4 & .0 & 6.2 & 9.0 & .5 \\
\hline .0 & .0 & .0 & .0 & .0 & .3 & .0 & 2.8 & .0 & .0 \\
\hline .0 & .0 & 7.7 & .0 & .0 & .1 & .0 & .7 & .0 & .0 \\
\hline .0 & .0 & .0 & .0 & .0 & .0 & .0 & .0 & .0 & .0 \\
\hline .0 & .0 & .0 & .0 & .0 & .0 & .0 & 7.2 & .0 & .0 \\
\hline .0 & 13.1 & .0 & .0 & 12.3 & .7 & .0 & 8.8 & .0 & .0 \\
\hline .0 & 10.6 & 28.5 & .0 & 8.8 & 1.5 & .0 & 26.9 & .0 & .0 \\
\hline .0 & 1.0 & 4.0 & .0 & .6 & .0 & .0 & .0 & .0 & .0 \\
\hline .0 & 4.6 & 17.5 & .0 & 2.7 & .8 & .0 & 5.2 & .0 & .0 \\
\hline .0 & 7.9 & .0 & .0 & 4.7 & 2.3 & .0 & 50.2 & .0 & .0 \\
\hline .0 & .7 & .0 & .8 & 7.3 & 29.4 & 2.5 & 4.3 & .0 & 16.0 \\
\hline .0 & 1.2 & 15.5 & .0 & 5.6 & 3.2 & .0 & 9.5 & .0 & .0 \\
\hline .0 & .0 & .0 & .0 & .0 & 2.5 & .0 & 5.9 & .0 & .0 \\
\hline .0 & .0 & .0 & .0 & .3 & .9 & .0 & .1 & .0 & .0 \\
\hline .0 & 38.2 & 44.2 & .0 & 112.1 & 62.8 & .0 & 135.5 & .0 & .0 \\
\hline
\end{tabular}

$13 \quad 14 \quad 15$

$\begin{array}{rrr}.9 & .0 & 253.2 \\ 2.0 & 4.2 & 235.7\end{array}$

$\begin{array}{rrr}2.0 & 4.2 & 235.7\end{array}$

$\begin{array}{rrr}19.4 & .0 & 16.1 \\ .0 & .0 & 163.3 \\ .0 & .0 & 34.7\end{array}$

$\begin{array}{rrr}.0 & .0 & 34.7 \\ 35.9 & .0 & 44.8\end{array}$

77.8

.0

3.2
.0

.0
5.3

52.6

161.4

505.4

95.7
75.2

90.4

214.7

132.7

36.2

59.1

1.7
.0

5.6

1.8
16.9

40.0

33.6

55.8

11.5

1.5

$9.4 \quad 10.5$

.0

RIVER BASIN KEY

$\begin{array}{ll}1 \text { Blackwater } & 8 \text { Patuxent } \\ 2 \text { Chester } & 9 \text { Pocomoke } \\ 3 \text { Choptank } & 10 \text { Potomac } \\ 4 \text { Gunpowder } & 11 \text { Rappahannock } \\ 5 \text { James } & 12 \text { Wicomico } \\ 6 \text { Nanticoke } & 13 \text { York } \\ 7 \text { Patapsco } & 14 \text { Elk }\end{array}$

15 Chesapeake Bay and Atlantic Ocean 
TABLE 2 (continued)

Industry Outputs and Population in 1970 by Major River Basins within the Chesapeake Bay Region (Outputs are in Millions of 1970 Dollars, Population is in Thousands)

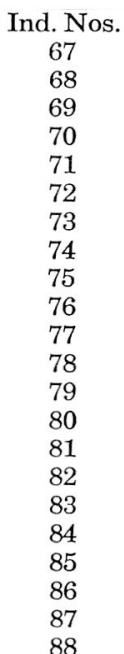

Population
RIVER BASINS

6

7

85

910

11

12

13

14

15

1.2
.0
.0
.0
.0
.0
.0
1.5
9.1
3.3
.0
27.2
.0
.0
10.9
7.5
10.7
6.0
3.6
2.3
.7
9.9

$\begin{array}{rr}1.8 & 11.6 \\ .7 & .0 \\ 8.3 & 8.4 \\ 4.9 & .0 \\ 5.4 & 458.7 \\ 1.7 & 3.4 \\ .0 & 32.6 \\ 3.1 & 84.7 \\ 5.4 & 145.5 \\ 24.6 & 125.9 \\ 2.2 & 12.6 \\ .0 & 131.7 \\ .0 & 77.3 \\ 1.1 & 9.0 \\ 19.3 & 325.1 \\ 10.2 & 335.9 \\ 50.6 & 558.9 \\ 5.7 & 114.3 \\ 24.2 & 263.6 \\ 5.4 & 62.7 \\ 6.9 & 30.6 \\ 17.0 & 204.3\end{array}$

$\begin{array}{ll}\mathbf{1} & 2.1 \\ .0 & 9.1\end{array}$

$\begin{array}{rr}2.1 & 1.5 \\ .8 & .2 \\ 9.1 & \\ 5.4 & 2.8\end{array}$

1.5
.2
.5
2.8

.0
.0
.0
.0

29.5
0

149.3

131.1
9.5

$\begin{array}{ll}.7 & 7.5 \\ .0 & 4.3\end{array}$

.9
2.4

.0
2.5

.0
5.7
24.2

2.4
.1
.5

1.9
.0

9.5
14.0

14.0
28.9

12.7

$\begin{array}{lll}2.5 & 24.2 & 22.8 \\ .1 & 37.0 & 19.2\end{array}$

1.3

481.8

365.1

116.9

$12.5 \quad 3.3$

19.2

.3

268.7
423.1

$\begin{array}{rr}.0 & 1.8 \\ 2.5 & 46.3\end{array}$

8.4
6.2
5.8

6.2
5.8
67.5

.0
.2

29.8
1011.8

1011.8
736.7

5240.8

417.2

107.5

36.6
313.7

313.7

11.4
2.1

$1.2 \quad 19.1$

28.1
149.5

$\begin{array}{lr}52.2 & 149.5 \\ 128 & 20.9\end{array}$

\begin{tabular}{ll}
12.8 & 20.9 \\
11.3 & 21.1 \\
\hline
\end{tabular}

$1.5 \quad 2470.5$

$\begin{array}{rr}1.1 & 241.9 \\ .9 & 152.3 \\ 1.5 & 1761.7\end{array}$

152.3
1761.7

$13.6 \quad 3056.1$

2.6
.0
17.1
.0
3.3
.0
.0
.0
7.3
11.9
1.7
11.6
7.1
4.4
23.0
18.5
45.3
13.8
12.9
6.5
3.4
22.4

129.6

2.6
.0
17.1

.0
3.3

.0
.0

7.3

11.9

1.7

7.1
4.4

23.0

18.5
45.3

13.8

12.9

3.4
22.4

129.6

.0
.0
.0
.0
4.9
.0
.0
.0
6.1
8.9
4.0
18.7
.0
.1
16.0
12.3
17.7
5.6
6.6
4.2
2.7
14.5

.5
.0
.0
.0
2.6
2.6
.0
.0
18.0
7.1
5.3
26.7
.4
1.9
18.4
17.0
89.5
25.4
32.0
12.1
13.2
30.9

.0
0

5.1

$.0 \quad 316.2$

$\begin{array}{ll}12.6 & 54.9\end{array}$

$\begin{array}{rr}12.6 & 54.9 \\ 8.7 & 273.3\end{array}$

$12.3 \quad 273.0 \quad 439.8$

49.1

254.7

RIVER BASIN KEY

$\begin{array}{ll}1 \text { Blackwater } & 8 \text { Patuxent } \\ 2 \text { Chester } & 9 \text { Pocomoke } \\ 3 \text { Choptank } & 10 \text { Potomac } \\ 4 \text { Gunpowder } & 11 \text { Rappahannock } \\ 5 \text { James } & 12 \text { Wicomico } \\ 6 \text { Nanticoke } & 13 \text { York } \\ 7 \text { Patapsco } & 14 \text { Elk } \\ 15 & \text { Chesapeake Bay and Atlantic Ocean }\end{array}$


TABLE 3

Gross Residual Projection for Nitrogen for the Chesapeake Bay Region (tons)

State/River Basin:

District of Columbia

$$
\text { Potomac }
$$

Maryland

Blackwater

Chester

Choptank

Gunpowder

Nanticoke

Patapsco

Patuxent

Pocomoke

Potornac

Wicomico

Elk

Ches Bay and Ocean

$$
\text { Total }
$$

Virginia

James
Potomac
Rappahannock
York
Ches Bay and Ocean

$$
\text { Total }
$$

Years

1970

4032.7

487.2
4696.4
10721.2
4462.4
3088.9
6309.8
12641.5
6802.5
47979.5
8067.9
3898.1
30092.2
139247.4

1975

1980

1985

Average Annual

Growth Rate

1970 to 1985

4252.4

4746.8

5214.8

1.7

525.0

5526.3

11868.7

5029.4

3340.6

6839.7

13502.7

7291.6

50891.9

8624.9

4460.8

32602.6

150504.0

29956.5

15088.8

12799.8

10840.1

12592.4

81277.6

$\begin{array}{rr}48850.0 & 79234.1 \\ 23024.4 & 35782.5 \\ 22386.9 & 38444.0 \\ 17875.6 & 29481.1 \\ 16292.5 & 21398.4 \\ 128429.4 & 204340.0\end{array}$

$\begin{array}{rrr}542.5 & 551.6 & .8 \\ 6336.1 & 6876.6 & 2.6 \\ 12816.9 & 13440.2 & 1.5 \\ 5400.6 & 5560.9 & 1.5 \\ 3495.8 & 3617.2 & 1.1 \\ 7139.6 & 7212.5 & .9 \\ 14078.7 & 14421.6 & .9 \\ 7521.9 & 7772.2 & .9 \\ 52343.6 & 52482.8 & .6 \\ 8875.6 & 9145.5 & .8 \\ 5069.7 & 5575.7 & 2.4 \\ 34425.1 & 35634.6 & 1.1 \\ 158045.9 & 162291.4 & 1.0\end{array}$

$\begin{array}{rr}122084.0 & 9.8 \\ 53028.2 & 8.7 \\ 62038.6 & 11.1 \\ 46196.8 & 10.1 \\ 27888.4 & 5.4 \\ 311235.8 & 9.4\end{array}$


TABLE 4

Gross Residual Projection for Biochemical Oxygen Demand for the Chesapeake Bay Region (tons)

State/River Basin:

District of Columbia

$$
\text { Potomac }
$$

Maryland

Blackwater

Chester

Choptank

Gunpowder

Nanticoke

Patapsco

Patuxent

Pocomoke

Potomac

Wicomico

Elk

Ches Bay and Ocean

$$
\text { Total }
$$

Virginia

James

Potomac

Rappahannock

York

Ches Bay and Ocean

Total
Years

$$
1970
$$

38612.9

729.4

10281.4

31769.8

14968.8

8747.0

25776.3

32344.2

21823.9

176318.4

27709.6

11005.3

141073.4

502547.4

154138.3

72888.0

49634.6

44275.0

49726.6

370662.3
1980

50787.8

748.5

9550.1

31255.7

14835.0

8577.6

26450.5

33504.4

20116.2

172217.5

26948.4

9985.7

154786.3

508975.8

371050.6

158841.0

149618.2

121644.6

86832.0

887985.9
Average Annual

Growth Rate

1970 to 1985

$$
1985
$$

58567.2

$\begin{array}{rr}715.7 & -.1 \\ 8606.0 & -1.2 \\ 29310.8 & -.5 \\ 14156.2 & -.4 \\ 7999.9 & -.6 \\ 25899.7 & .0 \\ 33425.2 & .2 \\ 17956.2 & -1.3 \\ 163285.9 & -.5 \\ 24975.7 & -.7 \\ 9026.7 & -1.3 \\ 158550.1 & .8 \\ 493908.0 & -.1\end{array}$

550638.6

228516.9

241168.4

189262.8

113468.8

1323054.9 
extends in a long, narrow band southeastward through Central and Southern Maryland to the Chesapeake Bay.

The Patuxent River was selected as the subject for the water quality management simulation for several reasons. First, the hydrologic information required for the development of transfer coefficients was available from past and ongoing research on this basin. ${ }^{\top}$ Projections of future wastewater flows to existing and proposed waste treatment plants were also available. In addition, the $\mathrm{Pa}$ tuxent River basin was selected for the management simulation because of its ecologically vulnerable position between the rapidly expanding metropolitan areas of Washington, D.C., Annapolis, and Baltimore, Maryland.

The water quality management model developed for the simulation experiment has several important characteristics that distinguish it from other water quality models. ${ }^{8}$ First, the model is designed to consider simultaneously several waste residual types and several water quality parameters. Interdependencies or synergisms among waste residuals during and after treatment are also considered. These interdependencies are explicitly recognized within the management model by "joint-product" waste discharge and treatment cost functions, and multiple residual transfer coefficient sets.

A second distinguishing characteristic of the management model is the functional form of the waste treatment cost functions. These piecewise linear convex functions are derived from polynomials fit to the latest information available on conventional and advanced waste-water treatment costs. A final distinguishing characteristic of the management model is that its components facilitate an examination of both the incentive mechanisms for the decentralization of decision making throughout the Chesapeake Bay region and the "tatonnement" procedures required for the implementation of these mechanisms when cost or hydrologic information is lacking.

In order to employ the management model within the Patuxent River basin, the River was divided into twenty-two distinct segments called "reaches". Each reach was assigned a number. Reaches 20,21 , and 22 , located at the heads of the river and its two primary tributaries, are "dummy reaches" designed to represent the given, or initial, water quality conditions throughout the management area of the river. Water quality in reaches one through nineteen, measured by the dissolved oxygen (D.O.) concentration in milligrams per liter $(\mathrm{mg} / \mathrm{l})$, is dependent on the hydrologic parameters of the river, the natural additions along the river, the water quality in the "dummy reaches", and the quantity of biochemical oxygen demand and nitrogen discharged to the river from waste handling activities. ${ }^{9}$ Fixed transfer coefficient sets, one for biochemical oxygen demand and another for nitrogen, are used in the model to represent the marginal effects of unit additions of biochemical oxygen demand or nitrogen on downstream water quality.

Year 2000 waste water flows are employed in the water quality management simulation for the Patuxent River basin. These flows are confined to domestic waste sources and thus to publicly owned treatment plants. ${ }^{10}$ 
Present and projected waste water flows for the nine waste treatment plants located within the Patuxent River basin management area are provided in Table 5. The projected five-fold increase in waste water flows over this and the next two decades provides a simple index of development pressure on the assimilative resources of this river. Table 5 also defines each plant location by Maryland county and reach number.

The primary objective assumed throughout the management simulation is to satisfy a water quality (river) standard throughout the Patuxent Basin at minimum total treatment costs, subject to program constraints. ${ }^{11}$ The program constraints define the conditions under which the river basin authority charged with satisfying the standard may allocate treatment responsibility and cost among the individual plants.

Four management programs were simulated throughout the basin, each program specified by a particular program constraint. Three of the management programs rely on "effluent charges", or taxes, to satisfy the water quality standard. For each of these incentive programs, the program constraint is defined by the information available to the river basin authority on waste treatment cost and river hydrology. The fourth program relies on equal percentage treatment at the nine waste treatment plants. ${ }^{12}$

The year 2000 water quality management simulation for the Patuxent River was based on mean August flow conditions of the river and a dissolved oxygen standard of four milligrams per liter. Financial conditions selected for the simulation comprise an interest rate of six percent, an ammortization period of twentyfive years, and no federal or state participation in plant capital expenditures.

\section{Minimum Cost Effluent Charges:}

Minimum cost effluent charges are taxes which when levied on untreated plant effluent will elicit the treatment pattern, or level of residual removal at each plant, that satisfies the water quality standard at minimum total cost. These charges, which reflect differences in both the treatment cost parameters among plants and the assimilation capacity among reaches, are determined from the optimal solution of the linear programming problem specified to allocate treatment responsibility among the nine dischargers to achieve the standard in the nineteen reaches at minimum total cost. ${ }^{13}$

Table 6 lists residual discharge and cost information for the nine waste treatment plants under each management plan. Plant totals are also provided. The total treatment cost per day for the nine plants under the minimum cost effluent charge program is $\$ 28,278$. The total daily effluent charge payments collected by the river basin authority are equal to $\$ 8,982$. Daily residual discharges to the Patuxent River are equal to 3,562 and 12,340 pounds of biochemical oxygen demand (BOD) and nitrogen respectively.

\section{Weighted Effluent Charges:}

When treatment cost information is not available for the waste treatment plants, weighted effluent charges can be employed under a "tatonnement" pro- 
TABLE 5

WASTE TREATMENT PLANT INFORMATION

\begin{tabular}{|c|c|c|c|c|}
\hline \multirow[t]{2}{*}{ Plant Service Area Name } & \multicolumn{2}{|c|}{ Plant Location } & \multicolumn{2}{|c|}{$\begin{array}{c}\text { Estimated Average Wastewater Flows } \\
\text { Collected for Treatment* } \\
\text { (millions of gallons per day) }\end{array}$} \\
\hline & County & Reach No. & 1970 & 2000 \\
\hline Savage & Howard & 2 & 1.56 & 25.10 \\
\hline Md. House of Corrections & Anne Arundel & 1 & .60 & 1.20 \\
\hline Fort Meade Number 1 & Anne Arundel & 6 & 2.12 & 3.36 \\
\hline Fort Meade Number 2 & Anne Arundel & 5 & 1.53 & 2.44 \\
\hline Patuxent & Anne Arundel & 7 & 1.85 & 9.75 \\
\hline Maryland City & Anne Arundel & 8 & .70 & 11.98 \\
\hline Laurel Parkway & Prince Georges & 9 & 4.06 & 15.00 \\
\hline Bowie-Horsepen & Prince Georges & 15 & .75 & 1.75 \\
\hline Bowie-Belair & Prince Georges & 16 & 2.40 & 4.00 \\
\hline Totals & & & 15.57 & 74.58 \\
\hline
\end{tabular}

*Source: Maryland Environmental Service. 
cess to achieve a "second best" solution (from an efficiency standpoint) for the assignment of treatment responsibility within the Patuxent River basin. This type of effluent charge retains the transfer coefficient "weights" used to determine the minimum cost charges while simplifying the form of the "shadow price" to which each weight is applied. In this instance the "shadow price" is assumed to be constant for all reaches. ${ }^{1+}$ Residual discharge and cost information for the nine waste treatment plants under the weighted effluent charge program is provided in Table 6 .

\section{Uniform Effluent Charges:}

When neither waste treatment cost information nor residual transfer coefficients are available within a river basin, decentralized decision making that leads to program compliance can be elicited through a system of uniform effluent charges. In this instance the effluent charge is a single per-unit tax levied on all oxygen demanding residuals discharged to the Patuxent River. ${ }^{15}$

A "tatonnement" procedure similar to that employed under the weighted effluent charge program is used to find the minimum tax required for the satisfaction of the water quality standard. Treatment plant discharges and costs under this management program are recorded in Table 6.

\section{Equal Percentage Treatment:}

This final management program relies on environmental fiat rather than monetary incentives to obtain the satisfaction of water quality standards. Satisfaction of the four milligram per liter standard for dissolved oxygen in the nineteen reaches of the Patuxent River requires that 98.9 percent of the biochemical oxygen demand and 97.0 percent of the nitrogen in waste influent be removed at each of the nine treatment plants in the year 2000. Treatment plant discharges and costs are estimated in Table 6.

\section{The Management Programs Compared:}

The four alternative water quality management programs for the Patuxent River can be compared as to plant treatment costs, effluent charge revenue generation, waste treatment and discharge levels, information requirements, and ease of administration. Several of these comparisons can be made on the basis of the information contained in Table 6.

The waste treatment cost (cost at plant) totals can be employed to measure the efficiency of each management program in satisfying the dissolved oxygen standard along the nineteen reach segment of the Patuxent River in the year 2n00. The minimum cost program is, by definition, the most efficient of the four management programs. Total daily costs are 6.1 percent higher under the weighted charge program, 22.2 percent higher under the uniform charge program, and 29.0 percent higher under equal percentage treatment. For any management program the distribution of the treatment costs among the nine plants, and thus the equity of the program, can be examined in Table 6 . However, for any specific 
management program, an examination of the fairness, or equity, of the treatment allocation must necessarily falter for the same reasons that invalidate interpersonal welfare comparisons.

If the effluent charge revenue collected by the river basin authority is returned to the Patuxent basin, perhaps in the form of research payments or expenditures for flow or plant scale augmentation, the tax payments represent a pure transfer with respect to basin-level economic welfare. It follows that the aggregate measure of basin welfare, namely economic efficiency, is maximized under the minimum cost effluent charge program and minimized under the equal percentage treatment program. However, if the plants are considered as individual economic decision units the effluent charge payments represent real costs to the plant owners not unlike the plant waste treatment expenditures. In this instance the water quality management programs preferred by individual treatment plants will not coincide necessarily with the minimum cost program that maximizes basin-wide aggregate economic welfare.

The information presented in Table 6 can be used to illustrate this latter point. The total expenditure at any plant required under a particular management program is equal to the sum of the plant treatment cost and the effluent charge payments made to the river basin authority. For any plant these total expenditure figures can be employed to rank the management programs as to economic burden. A rank of one is assigned to the program with the smallest required expenditure; a rank of four is assigned to the program with the largest required expenditure. This total expenditure rank is denoted for each plant by. a number enclosed by brackets in Table 6. Notice that six of the nine plant owners would prefer the minimum cost effluent charge program over the other three water quality management programs. Three plant owners would prefer equal percentage treatment. Eight of the nine plant owners suffer the greatest economic burden under the management program that employs uniform effluent charges. ${ }^{16}$

The total revenue generated by each of the three management programs that employ effluent charges is listed in Table 6 . The basin authority for the Patuxent River collects the greatest revenue under the weighted charge program and the least revenue under the uniform charge program.

The total quantities of biochemical oxygen demand (BOD) and nitrogen discharged to the Patuxent River under each water quality management plan are also listed in Table 6 . Notice that total discharge is highest (and thus overall treatment lowest) under the minimum cost program. The equal percentage treatment program is shown to be the least efficient management alternative in the use of the assimilative resources of the River.

The information requirements of the four management programs are quite diverse. The achievement of the water quality standard with minimum cost effluent charges requires information sufficient to specify all waste treatment cost and residual transfer functions. Weighted charges require only the residual transfer functions. Uniform charges and equal percentage treatment require no information other than the continuous dissolved oxygen measurements required under all management programs. 
Other information is required for program administration. A system of continuous metering devices must be installed within treatment plant outfalls under those management programs that rely on effluent charges for plan compliance. The equal percentage treatment program requires either effluent metering or periodic equipment inspection for plan compliance. Although there is no way to estimate the administrative costs of these management programs, the information requirement, and perhaps the information costs, under each program is roughly proportional to the efficiency of the program.

Although the water quality management simulation was based on residual discharge and hydrologic conditions specific to the Patuxent River basin, many of the general results are also pertinent to the economics of water quality management within the total Chesapeake Bay region. The use of decentralized incentive mechanisms, or effluent charges, for water quality management is highly desirable from the viewpoint of Bay region economic efficiency and equity. In terms of efficiency these charges can be employed to elicit that distribution of treatment responsibility among residual discharges that satisfies the water quality standard at a minimum total cost of treatment. In terms of economic equity within the Chesapeake Bay region the incentive property of these effluent charges assures that the marginal cost of treatment at each residual discharge point is equal to the marginal system cost (for a given level of information availability) of satisfying the water quality standard.

Very little can be said about the equity or fairness of one management plan in comparison with another in terms of treatment distribution and cost. Such comparisons involve interpersonal, and in this instance intra- and interjurisdictional, welfare comparisons. However, the information displayed in Table 6 indicates that a switch within the Patuxent River basin from uniform charges to weighted charges or a switch from weighted charges to minimum cost charges should be welcomed by all residual dischargers.

This system of progressive movement from lesser to more efficient decentralized incentive programs lends itself well to water quality management within the Chesapeake Bay region, a region lacking the detailed treatment cost and hydrologic information required for the immediate implementation of either minimum cost or weighted effluent charge programs within its many river basins. As more treatment cost and hydrologic information becomes available for the Bay region in the future, perhaps from research funded by pilot uniform charge programs, the water quality management program can be altered to employ in a progressive fashion the weighted and minimum cost effluent charge incentive systems.

This study has illustrated, using both macro- and micro-economic-environmental approaches, that the maintenance of conservative levels of water quality within the Chesapeake Bay region in the future will require very high levels of tertiary liquid waste treatment, even under conditions described as "optimal" with respect to the spatial distribution of treatment plant scale and waste removal efficiency. The cost of this treatment in terms of the regional economic resources that must be committed to this purpose is immense. 
Waste Treatment Plant Discharges and Costs Under Alternative

Management Programs for the Patuxent River Basin in 2000*

(discharges in pounds per day, costs in dollars per day)

\begin{tabular}{|c|c|c|c|c|c|c|c|c|c|c|}
\hline \multirow[b]{2}{*}{$\begin{array}{l}\text { Management } \\
\text { Programs: }\end{array}$} & \multicolumn{9}{|c|}{ Waste Treatment Plants: } & \multirow[b]{2}{*}{$\begin{array}{l}\text { Total } \\
\text { all } \\
\text { plants }\end{array}$} \\
\hline & Savage & $\begin{array}{l}\text { Md. House } \\
\text { of } \\
\text { Corrections }\end{array}$ & $\begin{array}{c}\text { Fort Mead } \\
\text { No. } 1\end{array}$ & $\begin{array}{c}\text { Fort Mead } \\
\text { No. } 2\end{array}$ & Patuxent & $\begin{array}{l}\text { Maryland } \\
\text { City }\end{array}$ & $\begin{array}{c}\text { Laurel } \\
\text { Parkway }\end{array}$ & $\begin{array}{l}\text { Bowie- } \\
\text { Horsepen }\end{array}$ & $\begin{array}{l}\text { Bowie- } \\
\text { Belair }\end{array}$ & \\
\hline \multicolumn{11}{|l|}{ Minimum Cost Charges: } \\
\hline BOD discharged & 942 & 110 & 295 & 267 & 683 & 417 & 414 & 154 & 280 & 3,562 \\
\hline Nitrogen discharged & 2,764 & 485 & 1,282 & 1,217 & 2,789 & 1,142 & 850 & 667 & 1,144 & 12,340 \\
\hline Cost at plant & 8,591 & 529 & 1,172 & 833 & 3,011 & 5,109 & 6,774 & 714 & 1,545 & 28,278 \\
\hline Eff. charge payments & 2,109 (2) & $195(1)$ & 398 (1) & 398 (1) & 1,709 (1) & $1,623(2)$ & $1,250 \quad(2)$ & $389(1)$ & $703(1)$ & 8,982 \\
\hline \multicolumn{11}{|l|}{ Weighted Charges: } \\
\hline BOD discharged & 873 & 105 & 235 & 171 & 511 & 417 & 363 & 123 & 280 & 3,079 \\
\hline Nitrogen discharged & 2,393 & 458 & 961 & 698 & 1,859 & 1,142 & 572 & 501 & 1,144 & 9,728 \\
\hline Cost at plant & 8,852 & 539 & 1,353 & 1,067 & 3,579 & 5,109 & 7,132 & 828 & 1,545 & 30,003 \\
\hline Eff. charge payments & 2,518 (3) & $311 \quad(2)$ & 789 (2) & $548(2)$ & $1,331 \quad(2)$ & 1,657 (3) & 923 & $400(2)$ & $744(2)$ & 9,220 \\
\hline \multicolumn{11}{|l|}{ Uniform Charges: } \\
\hline BOD discharged & 607 & 63 & 117 & 85 & 339 & 417 & 363 & 79 & 139 & 2,210 \\
\hline Nitrogen discharged & 957 & 229 & 320 & 233 & 930 & 1,142 & 572 & 267 & 381 & 5,031 \\
\hline Cost at plant & 10,366 & 749 & 1,975 & 1,553 & 4,364 & 5,109 & 7,132 & 1,075 & 2,243 & 34,566 \\
\hline Eff. charge payments & 1,705 & $318 \quad(3)$ & $477(4)$ & 346 (4) & $1,383(4)$ & 1,699 & 1,019 (4) & $378(4)$ & $567(4)$ & 7,893 \\
\hline \multicolumn{11}{|l|}{ Equal Percent Treatment: } \\
\hline BOD discharged & 694 & 33 & 93 & 68 & 270 & 331 & 415 & 48 & 111 & 2,063 \\
\hline Nitrogen discharged & 1,426 & 68 & 191 & 139 & 554 & 681 & 852 & 99 & 227 & 4,238 \\
\hline Cost at plant & 9,871 (1) & ) 1,076 (4) & $2,272 \quad(3)$ & 1,799 (3) & $4,940 \quad(3)$ & 5,749 & 6,771 (1) & 1,415 & 2,578 & 36,471 \\
\hline
\end{tabular}

*based on the waste treatment plant flows for the year 2000 in Table 5, a dissolved oxygen river standard of 4 milligrams per liter, an interest rate of $6 \%$, an ammortization period of 25 years, and no federal or state participation in plant construction expenses.

()$=$ total expenditure rank $(1-4)$ of the management programs from the viewpoint of the plant owner. Total expenditure $=$ cost at plant plus effluent charge payments. A rank of 1 is assigned to the management program with the smallest required total expenditure; a rank of 4 is assigned to the program with the largest required total expenditure. 
Major attention should be given to the alternative systems for liquid waste handling now operating on an experimental basis throughout the world. As development pressure on the assimilative resources of the Chesapeake Bay region continues in the future, these new systems of waste handling, including land treatment and non-water conveyance, should be fully investigated to determine the feasibility for their application throughout the Bay region.

Even with advanced water treatment technology and efficient application of financial incentives, the problem of protecting water quality through the 1980's will present such formidable technical, economic, aesthetic, and administration problems that additional more direct measures, such as land use planning to reduce rates of economic growth should also be considered to protect the Patuxent River and other components of the Chesapeake Bay system.

\section{FOOTNOTES}

1. Robert J. Korbach, An Environmental Linkages Model of the State of Maryland, Prepared for the Maryland Department of State Planning by thethe Bureau of Business and Economic Research, University of Maryland (College Park, Maryland), June, 1972.

2. These forecasts were provided by Professor Curtis C. Harris of the Regional Forecasting Project of the Bureau of Business and Economic Research at the University of Maryland.

3. Information on irrigated acreage at the county level was obtained from the 1969 Census of Agriculture, U.S. Department of Commerce, Bureau of the Census (Washington, 1972).

4. The information for minor civil divisions was obtained from the 1970 U.S. Census of Population.

5. For water residual " $\mathrm{k}$ " and minor civil division " $\mathrm{j}$ ",

$$
w_{j, k}=\sum_{i=1}^{94} r_{i, k} X_{i, j}
$$

where: $w_{j, k}$ = total residual of type " $k$ " generated in minor civil division " $\mathrm{j}$ ",

$\mathrm{r}_{\mathrm{i}, \mathrm{k}}=$ gross residual coefficient for activity " $\mathrm{i}$ " (92 industry outputs, population, or irrigated acreage) and water residual "k",

$\mathrm{X}_{\mathrm{i}, \mathrm{j}}=$ magnitude of activity " $\mathrm{i}$ " (92 industry outputs, population, or irrigated acreage) in minor civil division " $\mathrm{j}$ ".

6. The information for this segment of paper was extracted from Chapter VI of "The Economics of Regional Water Quality Management (A Case Study of River Water Quality in the Chesapeake Bay Region)" by Henry W. Herzog, Jr., (unpublished Ph.D. dissertation, Department of Economics, University of Maryland, 1974).

7. These coefficients provide a mathematical approximation of the second component of the "water quality problem".

8. The water quality management model is developed in Chapter V, Herzog, loc. cit.

9. Thus, the third component of the "water quality problem", environmental quality, (represented in this instance by the dissolved oxygen concentration) is intimately related to each of the other components.

10. The domestic waste sources account for roughly ninety-seven percent of the biochemical oxygen demand and eighty-five percent of the nitrogen residuals discharged within the basin.

11. This water quality standard is expressed in terms of the minimum level of dissolved oxygen, in milligrams per liter $(\mathrm{mg} / \mathrm{l})$, required in each reach.

12. Other programs considered in the research, but not reported here, concern the scale economies achieved with centralized waste treatment.

13. For the i-th treatment plant the effluent charges can be represented as:

$$
\begin{aligned}
& { }^{t_{i} \text { BOD }}=\sum_{j=1}^{22} \text { BOD }{ }^{\mathrm{i}, j} \Pi_{j} \\
& t_{\mathrm{i}} \text { NITC }=\sum_{\mathbf{j}=\mathbf{1}}^{22 \mathrm{NIT}} \mathrm{i} \mathbf{j} \Pi_{\mathbf{j}}
\end{aligned}
$$


where: $t_{i, B O D}$ and $t_{i}$ NIT are the per-unit charges on biochemical oxygen demand and nitrogen respectively, a ${ }_{i, j}$ and a ${ }_{i, j}$ are the transfer coefficients for biochemical oxygen demand and nitrogen respectively between reaches " $\mathrm{i}$ " and " $\mathrm{j}$ ", and $\Pi \mathrm{j}$ is the simplex multiplier for the $\mathrm{j}$-th reach ( $\mathrm{j}$-th water quality constraint) from the optimal solution of the linear program. $\Pi j$ measures the marginal system (river basin) cost of water quality maintenance in the $\mathrm{j}$-th reach. $\Pi \mathrm{j}$ can also be interpreted as the "shadow price" of water quality impairment in the $j$-th reach.

14. For the $\mathrm{i}$-th treatment plant the effluent charges can be represented as:

$$
\begin{aligned}
& t_{i}^{B O D}=I I^{*} \sum_{j=1}^{22} a_{i, j}^{B O D} \\
& \text { NIT } 22 \text { NIT } \\
& t_{i}=I I^{*} \sum_{j=1}^{22} a_{i, j}
\end{aligned}
$$

where II* is the single "shadow price" determined under a "tatonnement" process.

15. For each of the treatment plants the effluent charge can be represented as:

$$
\mathrm{t}_{\mathrm{i}}^{\mathrm{BOD}}=\mathrm{t}_{\mathrm{i}}^{\mathrm{NIT}}=\mathrm{II}^{*}
$$

where II* is determined under a "tatonnement" process.

16. Notice that a switch from uniform charges to weighted charges or a switch from weighted charges to minimum cost charges would be welcomed by the nine treatment plant owners, and would also increase basin-wide aggregate welfare (economic efficiency). 